\title{
Beam loss distribution calculation and collimation efficiency simulation of a cooler storage ring in a heavy ion research facility
}

\author{
Peng Li, ${ }^{1,}$ ' Youjin Yuan, ${ }^{1}$ Jiancheng Yang, ${ }^{1}$ Min Li ${ }^{1,2}$ Xiaodong Yang, ${ }_{1}^{1}$ Ruishi Mao, ${ }^{1}$ Jun Meng, \\ Peiyong Jiang, ${ }^{1,2}$ Wenheng Zheng, ${ }^{1,2}$ Zhen Chai, ${ }^{1}$ Guozhu Cai, ${ }^{1}$ and Shaoming Wang ${ }^{1}$ \\ ${ }^{1}$ Institute of Modern Physics, Chinese Academy of Sciences, Lanzhou 730000, People's Republic of China \\ ${ }^{2}$ University of Chinese Academy of Sciences, Beijing 100049, People's Republic of China
}

(Received 18 November 2013; published 28 August 2014)

\begin{abstract}
The Heavy Ion Research Facility in Lanzhou is an ion cooler storage ring facility in China's Institute of Modern Physics. The beams are accumulated, electron cooled, accelerated, and extracted from the main cooler storage ring (CSRm) to the experimental ring or different terminals. The heavy ion beams are easily lost at the vacuum chamber along the CSRm when it is used to accumulate intermediate charge state particles. The vacuum pressure bump due to the ion-induced desorption in turn leads to an increase in beam loss rate. In order to avoid the complete beam loss, the collimation system is investigated and planned to be installed in the CSRm. First, the beam loss distribution is simulated considering the particle charge exchanged process. Then the collimation efficiency of the lost particles is calculated and optimized under different position and geometry of the collimators and beam emittance and so on. Furthermore, the beam orbit distortion that is caused by different types of errors in the ring will affect the collimation efficiency. The linearized and inhomogeneous equations of particle motion with these errors are derived and solved by an extended transfer matrix method. Actual magnet alignment errors are adopted to investigate the collimation efficiency of the lost particles in the CSRm. Estimation of the beam loss positions and optimization of the collimation system is completed by a newly developed simulation program.
\end{abstract}

DOI: 10.1103/PhysRevSTAB.17.084201

PACS numbers: 29.27.Eg, 41.85.Si

\section{INTRODUCTION}

The HIRFL-CSR complex consists of the main cooler storage ring (CSRm), Radioactive Ion Beam line (RIB) production and transfer line two (RIBLL2), experimental storage ring (CSRe), and experimental stations. The two existing cyclotrons, the Sector Focus Cyclotron and Separated Sector Cyclotron, at the Heavy Ion Research Facility in Lanzhou (HIRFL) are used as the injector system. The heavy ion beams from HIRFL are injected into the CSRm, then accumulated, electron cooled, and accelerated, before being extracted to the CSRe for internal target experiments and other physics experiments $[1,2]$. The overall layout of the HIRFL-CSR can be seen in Ref. [1]. Until now, the HIRFL-CSR has succeeded in accumulating and accelerating particles from protons to uranium ions and providing different beams for physics experiments such as the radioactive electron-capture experiment, isochronous mass spectrometer precise mass measurements in the CSRe, and particle therapy experiments [3].

\footnotetext{
*Corresponding author. lipeng@impcas.ac.cn

Published by the American Physical Society under the terms of the Creative Commons Attribution 3.0 License. Further distribution of this work must maintain attribution to the author $(s)$ and the published article's title, journal citation, and DOI.
}

In order to accumulate the beams to higher intensity to fulfill the requirements of physics experiments [4] and for better understanding of the dynamic vacuum pressure caused by the beam loss, an accelerator machine protection method such as the collimation system is considered to be installed in the CSRm. Furthermore, a project at an international accelerator facility, the High Intensity Heavy Ion Accelerator Facility (HIAF) project, has been proposed by the Institute of Modern Physics (IMP) [5]. This complex can accumulate the reference particle ${ }^{238} \mathrm{U}^{34+}$ to energy $1.2 \mathrm{GeV} / \mathrm{u}$ with intensity $5 \times 10^{11} \mathrm{ppp}$ (particles per pulse). Therefore, a collimation system will be tested in the current CSRm to establish the technical storage for the new accelerator complex HIAF. Meanwhile, the future measurement results of the collimation system in the CSRm will be the benchmark of the newly developed program package.

According to the design requirements of the Facility for Antiproton and Ion Research (FAIR) project [6] at GSI Helmholtzzentrum für Schwerionenforschung $\mathrm{GmbH}$ in Germany, high beam intensities can be reached at the lower charge states of heavy ions since the space charge limit after injection into synchrotrons scales with the ratio of $A / q^{2}$, where $A$ is the atomic mass number and $q$ is the charge of the particle. In case of uranium beams, the intensities of heavy ion beams per synchrotron pulse is increased by the factor 6.8 by using the intermediate charge state $28+$ instead of $73+[6]$. But intermediate charge state particles are much easier lost when they collide with the residual gas atoms 


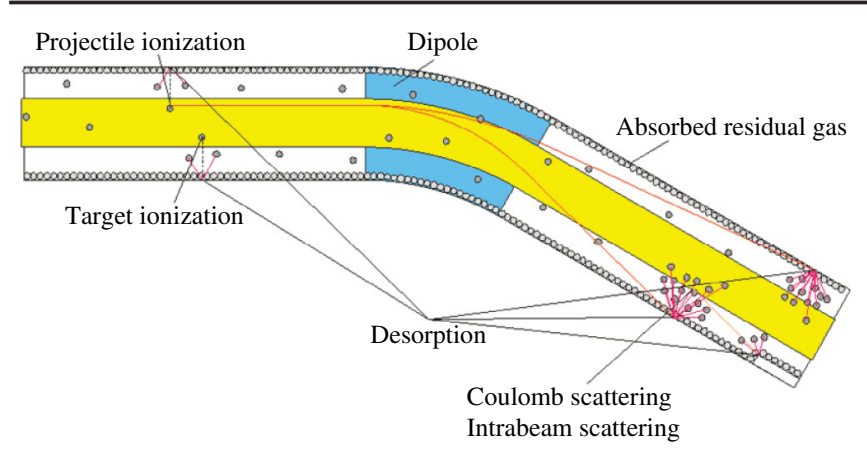

FIG. 1. Beam loss processes and interaction with the residual gas in an accelerator [9].

because they change to other charge states. The resulting change in the mass over charge ratio $m / q$ leads to modified trajectories in dispersive beam transport elements, and finally to the loss of the particle at the vacuum chamber [7]. The secondary particles are produced at the impact position by ion-induced desorption and as a result the pressure in the vacuum chamber is increased locally. This local rise in pressure in turn leads to increased charge changing processes starting an avalanche process that may eventually lead to a complete loss of the beam during a few turns in the synchrotron [8]. The beam loss processes in the accelerator is illustrated in Fig. 1 [9].

In order to suppress and control the gas desorption process, specifically designed beam catchers (collimators) with lower desorption rate material are installed at the impact positions [6-8]. The collimation efficiency, which is defined as the beam loss on the collimators divided by the total loss number, is used to evaluate the beam loss rate [7-9]. Collimation efficiency optimization aims to let all the lost particles hit on the catchers. In an accelerator, there are many factors, such as collimators' positions, geometry, beam emittance, electron cooling, and beam orbit distortion, that will affect the collimation efficiency.

The purpose of this paper is to present the study of the optimization of the collimation efficiency under different factors, limited machine space and fixed lattice structure. The calculation of beam loss position can determine the position and mechanical design of the collimators. The maximum collimation efficiency is expected to provide the theory basis for test and installation of the collimators.

\section{CSRm}

CSRm is a racetrack shape synchrotron that consists of four arc sections with the circumferences of $161.00 \mathrm{~m}$. Each arc section is composed of four dipoles, five focusing quadrupoles, and three defocusing quadrupoles. The lattice of each arc section is given as follows:

$$
-L 1-F D F-B-B-F-L 2-D F-B-B-F-\frac{1}{2} D,
$$

where $F$ is the focusing quadrupole and $D$ is the defocusing quadrupole in the horizontal direction, $B$ is the bending

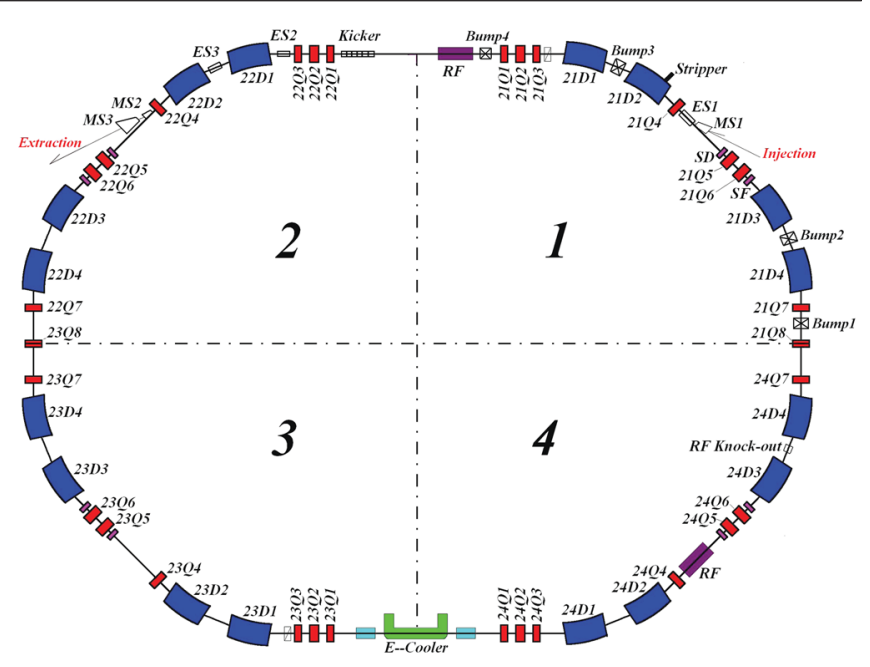

FIG. 2. The overall layout of the CSRm.

dipole, $L 1$ is a long-straight section with dispersion free for e-cooler, extraction kicker, or internal target, $L 2$ is a dispersion drift for beam injection, extraction, and rf cavity [10]. Figure 2 shows the global layout of the CSRm. The CSRm is divided into four quadrants in the plane coordinate system. In Fig. 2, blue sectors are $22.5^{\circ} \mathrm{H}$-type bending dipole magnets; red rectangles are quadrupole magnets; and pink rectangles are sextupole magnets [sextupole defocusing magnets (SD) or sextupole focusing magnets (SF)]. There are also some other elements, such as radio frequency cavity (rf), electrostatic septum (ES), magnetic septum (MS), kicker, stripper, and electron cooler (e-cooler).

A typical beam production cycle is as follows: injection, accumulation, capture, acceleration, harmonic change; for the second capture, next acceleration, slow or fast extraction. Because of the rf cavity frequency limitation, the $\mathrm{rf}$ harmonic number sometimes needs to be changed for the second capture during the acceleration cycle. According to the sequences mentioned above, there are four energy settings: the injection, mid flat top, extraction, and final. The CSR power supply data cycles are calculated according to the acceleration schedules, accelerator physics simulation, and requirements of the experiment [2]. The collimation system is designed for the beam injection energy. The positions of collimators can be adjusted during different machine cycling. Main parameters of the CSRm can be seen in Table I [11]. There are two methods to extract the beam from the CSRm: slow extraction and fast extraction [11]. The CSRm dipole magnetic field cycle for the reference beam uranium ${ }^{238} \mathrm{U}^{32+}$ is shown in Fig. 3. Detailed parameters for the ${ }^{238} \mathrm{U}^{32+}$ are illustrated in Table II in Sec. IV.

With large acceptance, two injection and accumulation methods are realized: one is the multiple multiturn injection (MMI) with electron cooling, which is designed for general accumulation; the other one is charge exchange injection with cooling designed for light nuclei. The major lattice parameters of the CSRm are shown in Fig. 4 [11]. The multiturn injection method, which is horizontal phase 
TABLE I. Basic parameters of the CSRm.

\begin{tabular}{|c|c|c|}
\hline CSRm & & \\
\hline Ion species & \multicolumn{2}{|c|}{ Stable nuclei: $\mathrm{P}-\mathrm{U}$} \\
\hline Maximum energy & \multicolumn{2}{|c|}{$2.8 \mathrm{GeV} / \mathrm{u}(\mathrm{P}), 1.1 \mathrm{GeV} / \mathrm{u}\left({ }^{12} \mathrm{C}^{6+}\right), 500 \mathrm{MeV} / \mathrm{u}\left({ }^{238} \mathrm{U}^{72+}\right)$} \\
\hline Intensity & \multicolumn{2}{|c|}{$10^{5}-10^{9} \mathrm{ppp}$ (stable nuclei) } \\
\hline Momentum spread & \multicolumn{2}{|c|}{$\Delta \mathrm{P} / \mathrm{P} \sim 10^{-4}(\mathrm{rms})($ stable nuclei after electron cooling) } \\
\hline Emittance (rms) (injection) & \multicolumn{2}{|c|}{$\varepsilon_{\mathrm{h}}(\pi \mathrm{mm}-\mathrm{mrad}) \leq 5($ stable nuclei $)$} \\
\hline \multirow[t]{4}{*}{ Acceptance } & \multirow{3}{*}{\multicolumn{2}{|c|}{$\begin{array}{c}\mathrm{A}_{\mathrm{h}}(\pi \mathrm{mm}-\mathrm{mrad})=200(\Delta \mathrm{P} / \mathrm{P}= \pm 0.3 \%) \\
\mathrm{A}_{\mathrm{v}}(\pi \mathrm{mm}-\mathrm{mrad})=40 \\
\Delta \mathrm{P} / \mathrm{P}=1.4 \%\left(\varepsilon_{h}=50 \pi \mathrm{mm}-\mathrm{mrad}\right)\end{array}$}} \\
\hline & & \\
\hline & & \\
\hline & Fast extraction mode & Slow extraction mode \\
\hline Transition gamma & $\gamma \operatorname{tr}=5.418$ & $\gamma \operatorname{tr}=5.168$ \\
\hline Tune values & $Q_{x} / Q_{y}=3.64 / 2.61$ & $Q_{x} / Q_{y}=3.63 / 2.61$ \\
\hline Natural chromaticity & $Q_{x}^{\prime} / Q_{y}^{\prime}=-3.17 /-5.37$ & $Q_{x}^{\prime} / Q_{y}^{\prime}=-3.05 /-5.34$ \\
\hline \multirow[t]{2}{*}{ Maximum $\beta$ amplitude } & $\beta \mathrm{x} / \beta \mathrm{y}=12.1 / 13.5 \mathrm{~m}$ (dipole) & $\beta \mathrm{x} / \beta \mathrm{y}=11.1 / 17.5 \mathrm{~m}($ dipole $)$ \\
\hline & $\beta \mathrm{x} / \beta \mathrm{y}=15.3 / 30.5 \mathrm{~m}($ quadrupole $)$ & $\beta \mathrm{x} / \beta \mathrm{y}=13.5 / 32.2 \mathrm{~m}$ (quadrupole) \\
\hline \multirow[t]{2}{*}{ Maximum dispersion } & $\mathrm{D} \max (\mathrm{x})=3.1 \mathrm{~m}($ dipole, $\beta \mathrm{x}=9.0 \mathrm{~m})$ & $\mathrm{D} \max (\mathrm{x})=3.2 \mathrm{~m}($ dipole, $\beta \mathrm{x}=10.4 \mathrm{~m})$ \\
\hline & $\mathrm{D} \max (\mathrm{x})=5.4 \mathrm{~m}($ quad, $\beta \mathrm{x}=9.9 \mathrm{~m})$ & $\mathrm{D} \max (\mathrm{x})=4.6 \mathrm{~m}($ quad, $\beta \mathrm{x}=8.0 \mathrm{~m})$ \\
\hline
\end{tabular}

space painting, employs an input septum unit with four programmed orbit bumps to paint the horizontal phase space in the CSRm [12]. In this paper, beam parameters for the MMI injection method, which is normally used to accumulate the heavy ion particles, are adopted.

\section{SIMULATION CODE}

In order to simulate the charge exchange driven beam loss and dynamic vacuum effects in heavy ion synchrotrons, GSI first developed a program package named StrahlSim during the past 10 years $[7,8]$. With the scientific collaboration between GSI and IMP, a new program package (ColBeam) designed for optimizing the collimation efficiency was developed by taking different types of errors into account in the accelerator. The verification of the StrahlSim physics model was performed by comparing simulation results with the experimental data. A very good agreement with the experimental data was achieved [7].

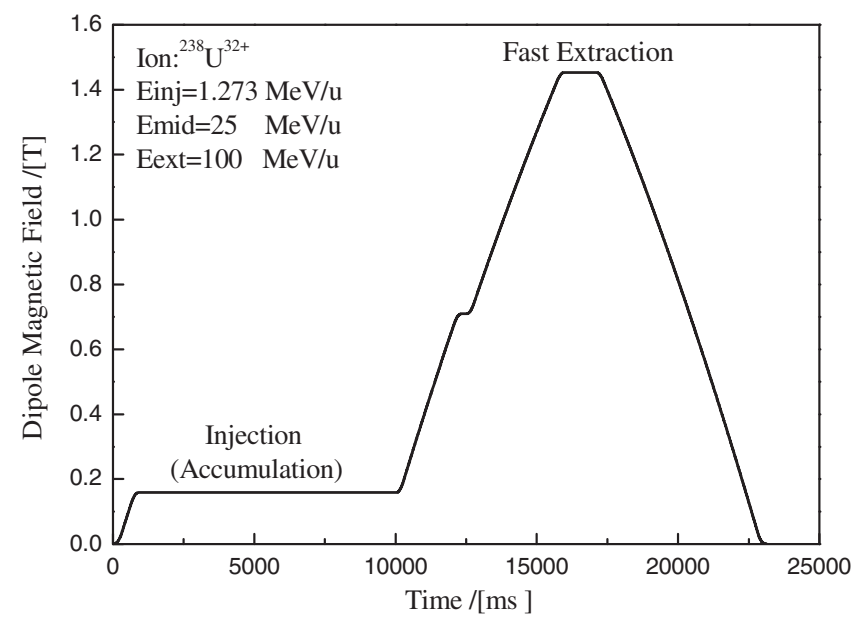

FIG. 3. Dipole magnetic field cycle for ${ }^{238} \mathrm{U}^{32+}$.
The calculation result of the ColBeam is verified by the StrahlSim [13].

The particles can be tracked in a ring during multiple turns or in a beam line just one-pass. First, the software package must load a lattice file that contains essential element parameters of the ring or beam line, such as element type, length, strength, vacuum chamber aperture, and so on. The lattice file with extension "LAT" for the simulation software Winagile [14] is used as the default input file. In this file, there are three types of vacuum chamber apertures: rectangular, elliptical, and superelliptical. The software reads the type of each element in the file and calculates the aperture according to the cross section equation. Users can adjust the element's position relative to the optical beam orbit, and then the aperture of the elements will be changed.

In order to obtain more accurate beam loss positions, this program package divides each lattice element into small pieces with a certain length. For example, in this simulation, the CSRm is discretized into elements with a length of about $10 \mathrm{~cm}$. Then the program package will draw the geometry of the ring or beam line according to the parameters loaded before. According to the element parameters, such as dipole

TABLE II. Simulation beam parameters.

\begin{tabular}{lc}
\hline \hline Reference ion & ${ }^{238} \mathrm{U}^{32+}$ \\
Energy $(\mathrm{MeV})$ & 1.273 \\
Transverse tune $(\mathrm{Qx} / \mathrm{Qy})$ & $3.605 / 2.61$ \\
Horizontal emittance $(\pi \mathrm{mm}$ mrad) $5 \sigma$ & 150 \\
Vertical emittance $(\pi \mathrm{mm}$ mrad) $5 \sigma$ & 25 \\
Longitudinal synchrotron period & 0.107 \\
Momentum deviation for ${ }^{238} \mathrm{U}^{32+}$ & $\sim 10^{-4}$ \\
Charge exchanged ion (coasting beam) & ${ }^{238} \mathrm{U}^{33+}$ and ${ }^{238} \mathrm{U}^{31+}$ \\
Vacuum chamber aperture $(\mathrm{h} / \mathrm{v}) \mathrm{mm}^{*} \mathrm{~mm}$ & \\
Dipole & $140 * 60$ \\
Quadrupole and drift & $160 * 100$ \\
\hline \hline
\end{tabular}




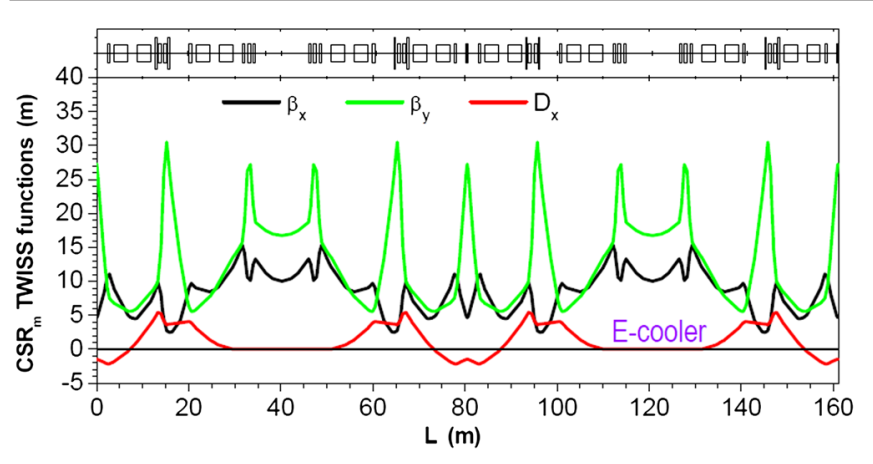

FIG. 4. Lattice parameters of the CSRm.

bending angle and length, quadrupole magnet gradient value and length, and drift length, the transfer matrix of each element can be calculated [15]. The twiss parameters can be calculated automatically by the program according to the transfer matrix of the ring or the beam line. A detailed calculation algorithm can be found in Ref. [15].

Beam envelope and dispersion function can be constructed and plotted in a graph window with the emittance, the number of sigma that defines the edge of the beam (this is the same for the two planes), and the full momentum spread of the beam with respect to the equilibrium orbit [15]. The beam envelope calculated by ColBeam is compared and agrees with the codes Winagile and StrahlSim. The effect of linear betatron coupling due to the skew quadrupole term and the solenoid will not be considered in this program package. Only the linear coupling produced by the quadrupole rotation errors is calculated. Six-dimensional transfer matrix is introduced to calculate the beam trajectory and an extended transfer matrix is implemented when considering different types of errors.

In this code, the normal accumulating beam is viewed as the reference ions and meanwhile, a certain number of charge exchanged particles can be generated in each small slice of the element according to the twiss parameters in the phase space. Each charge exchanged particle is presented as a six-coordinate vector: $\left(x, x^{\prime}, y, y^{\prime}, \Delta s, \Delta p / p\right)$, where $x$ and $y$ are the horizontal and vertical coordinates, $x^{\prime}$ and $y^{\prime}$ are the corresponding horizontal and vertical angles, $\Delta s$ is the distance from the bunch center (in the case of coasting beam-distance from a reference particle), and $\Delta p / p$ is calculated by Eq. (2) for the charge exchanged particles. The charge exchanged particle's initial coordinates are generated as Gaussian and truncated at $2 \sigma$ where $\sigma$ is the design rms value and with the centroid of the distributions equal to the design values. Each particle is generated according to the Gaussian random value and twiss parameters of each small slice of the element. In order to reduce the number of particles involved in the calculations, a macroparticle simulation method is adopted. Each of the macroparticles has a charge-to-mass ratio the same as a real particle, but the mass of the macroparticle is larger than the mass of the real particle by the factor of the particle increase [16]. The amplitude of the Gaussian function is viewed as the beam intensity factor for each macroparticle. The beam intensity factor is used to estimate the impact beam energy power on each collimator according to the total beam loss. The Gaussian distribution function is encoded by the classical formula [17]

$$
\omega=\sqrt{-2 \ln \omega_{1}} \cos \left(2 \pi \omega_{2}\right),
$$

where $\omega_{1}$ and $\omega_{2}$ are two independent random numbers that satisfy the uniform distribution (in the range from 0 to 1 ). The independent random number that has a Gaussian distribution with zero mean and a standard deviation of one is generated by Eq. (1). Different standard deviations of the beam Gaussian distribution function are calculated in this program.

For the charge exchanged particle, the deviation of the $\mathrm{m} / \mathrm{q}$ ratio of a particle with a different charge state $q$ compared to the reference ion with the charge state $q_{0}$ is equivalent to a momentum deviation $\Delta p / p$ of [7]

$$
\frac{\Delta p}{p}=\frac{q_{0}}{q}-1
$$

For example, ${ }^{238} \mathrm{U}^{32+}$ is chosen as the reference particle in this paper. The momentum deviation of one electroncapture particle ${ }^{238} \mathrm{U}^{31+}$ is $3.2 \%$ and one electron-loss particle ${ }^{238} \mathrm{U}^{33+}$ is $-3.03 \%$. There are two reasons for choosing uranium as the reference particle: intermediate charge state particles can be accumulated to higher beam intensity for the physics experiment; uranium is also chosen as the reference particle for the new facility (HIAF) [5].

All the charge exchanged particles are generated in the first turn. Multiple turn tracking for the charge exchanged particles will be implemented when these generated particles are not lost in one turn. During the simulation, the twiss parameters of particles that are not lost in one turn will be recorded and are viewed as the initial transfer parameters to be calculated during the next turns. The simulation will not terminate until all the generated particles are lost.

The loss positions and intensity factor of charge exchanged particles will be counted and stored when they collide with the collimators or their transverse beam size is larger than the vacuum chamber. The beam loss profile along the CSRm and collimation efficiency can be calculated after the tracking.

In order to simulate the effects of the beam orbit distortion on the collimation efficiency, this program package can read different files that contain the actual magnet alignment parameters and the ripple of the magnet power supplies. The extended transfer matrix to calculate the effect of the different types of errors can be found in Appendixes A, B, C, D.

\section{CSRm BEAM LOSS DISTRIBUTION}

For interactions with residual gas molecules, the electron-capture cross section dominates over the electron loss 


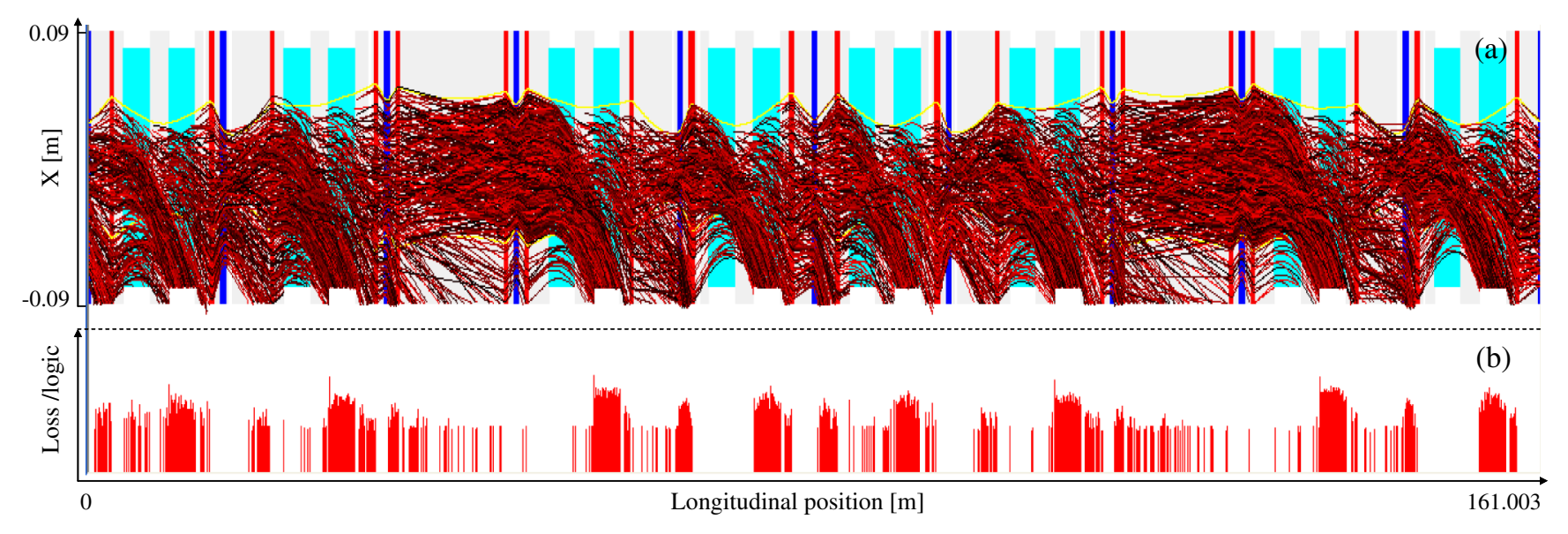

FIG. 5. Simulation results for one electron-loss ${ }^{238} \mathrm{U}^{33+}$ : (a) Beam loss trajectory. (b) Beam losses distribution. Loss/logic means: the logarithmic value of the lost beam intensity at each position.

at low collision energy. With the beam energy increasing, electron-capture cross sections decrease very rapidly due to a velocity mismatch, and then electron-loss processes begin to play a major role in charge-changing collisions [18]. The detailed data with respect to those two processes can be found in Ref. [18]. In addition, electron-capture processes occur in the electron cooler due to interaction with the high current electron beam [19]. In this paper, both electroncapture and electron-loss processes are simulated for the CSRm. The uranium beam ${ }^{238} \mathrm{U}^{32+}$ that was accumulated in the CSRm in 2011 is chosen as the reference ion to simulate the beam loss distribution with the machine operation parameters. The simulation beam parameters are listed in Table II.

With a constant vacuum pressure around the ring, the beam trajectory for one electron-loss ${ }^{238} \mathrm{U}^{33+}$ is illustrated in Fig. 5(a) and the beam trajectory for one electron-capture ${ }^{238} \mathrm{U}^{31+}$ is shown in Fig. 6(a). In the two figures, the red rectangle is the focusing quadrupole in horizontal and the blue rectangle is the defocusing quadrupole. The light blue rectangle is the dipole and the red line in the element is the charge exchanged particles' trajectory. It should be noted that some other element such as sextupole, kicker, and corrector and so on are replaced by simple drift space. The apertures of all the optical elements are rectangular of which the dimensions are listed in Table II. Each charge exchanged particle is a macroparticle with an intensity factor when it is generated. The number of lost particles at each divided slice are counted and the macroparticle intensity factors are added.

The loss position and intensity of the charge exchanged particle at each point is counted and shown in Figs. 5(b) and 6(b), respectively. A semilogarithmic coordinate system is employed to illustrate the beam loss intensity at each position along the CSRm. In Figs. 5(b) and 6(b), the $x$ axis is the longitudinal positions of the CSRm and the $y$ axis is the logarithmic value of the lost beam intensity at each position. The calculation method is $Y_{i}=\ln \left(I_{i} / I_{\text {total }}\right)$ where $I_{i}$ is the lost beam intensity at the $i$ position and $I_{\text {total }}$ is the total lost beam intensity of the whole ring. It is easier for the

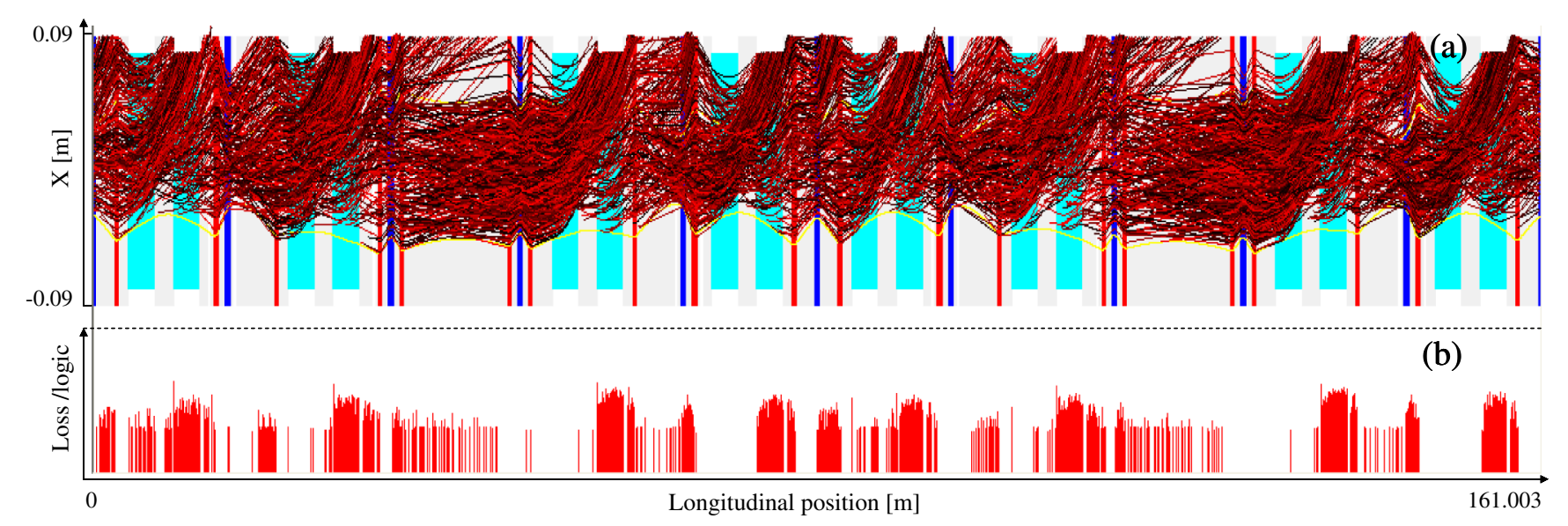

FIG. 6. Simulation results for one capture-loss ${ }^{238} \mathrm{U}^{31+}$ : (a) Beam loss trajectory. (b) Beam losses distribution. Loss/logic means: the logarithmic value of the lost beam intensity at each position. 


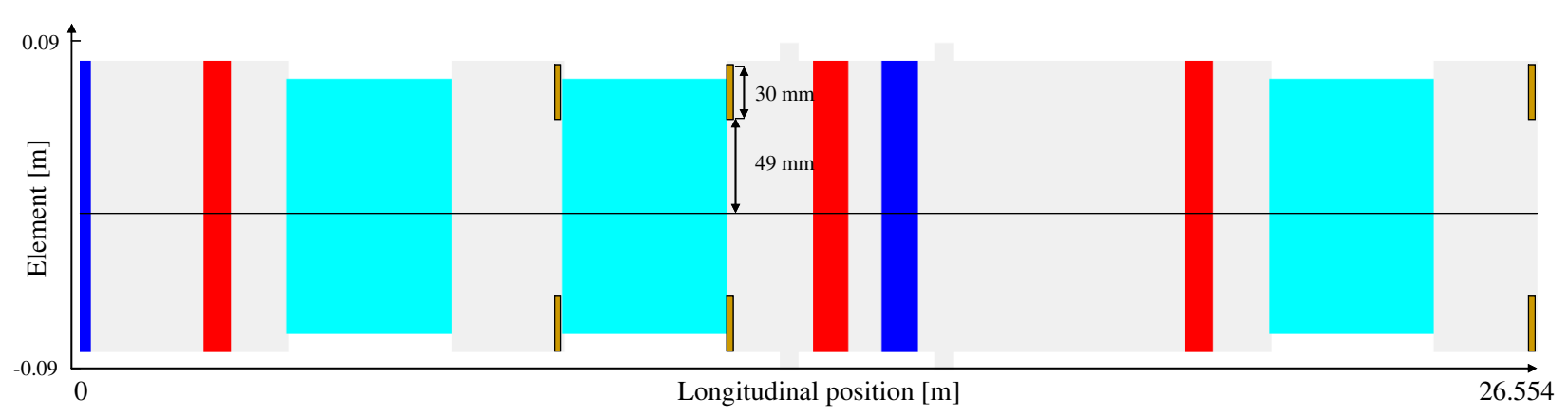

FIG. 7. Diagrammatic sketch of collimator geometry.

user to observe each lost point along the ring from this type of coordinate system.

The calculation shows that most of the charge exchanged particles are lost in the dipole or behind the dipole due to the dispersion function and the large momentum deviation. Without considering the beam loss induced by the charge exchange at the machine design stage, the losses cannot be concentrated at certain positions.

\section{COLLIMATION EFFICIENCY}

In order to avoid vacuum chamber desorption caused by the particle loss, several specially designed catchers (collimators) should be mounted "upstream" of the positions with high impact probabilities [7]. Most collimators are rectangle copper block with coated low-desorption material such as gold and nickel $[8,20]$. However, the length and positions of the collimators are limited by the actual mechanical structure in the compact CSRm. Some beam position monitors will be moved out to install the collimators [21].

For a detailed analysis of the performance for the collimation system, the intensity factor of particles hitting the collimators $N_{c}$ and the wall $N_{w}$ defines the collimation efficiency $\theta$ with [7]

$$
\theta=\frac{N_{c}}{N_{w}+N_{c}} .
$$

With higher collimation efficiency, most of the charge exchanged particles are lost on the collimators but not on the vacuum chamber to avoid the gas desorption. Generally, the collimation efficiency is the function of the beam emittance as well as the acceptance of the machine, the beam energy, the distance to the vacuum center, different standard deviations of the beam distribution, different beam distribution and lattice structure, and so on. The most important factor affecting the collimation efficiency is the structure of the lattice [22]. The lattice must provide the required space in each cell for the collimation system and ensure that practically no ions are lost elsewhere. This can be achieved if the lattice structure is optimized such that each lattice cell acts as a charge separator, providing a waist at the position of maximum separation [8]. Unfortunately, by design, the lattice of the CSRm is unalterable and each cell cannot be treated as a charge separator; therefore, the collimation efficiency only can be optimized based on the current lattice structure and the limited free space.

By means of removing some beam position monitors and correctors, only 21 collimators will be installed in the limited space. The diagrammatic sketch of its geometry and location can be seen in Fig. 7. The length of the collimator in the horizontal direction and the distance to the beam center will be optimized in the simulation. Meanwhile, the machine acceptance cannot be reduced during the collimator movement [21].

At the beginning of this project, only two prototype collimators are going to be tested in the CSRm first. The geometry of the collimator is calculated according to the beam energy range and intensity in the CSRm. The parameters of the collimator can be seen in Table III.

The one electron-loss particles' trajectory and the loss positions along the CSRm are illustrated in Fig. 8(a). In this picture, each collimator's length is $30 \mathrm{~mm}$ and locates at the beam envelope edge that is calculated with the emittance. Figure 8 (b) shows the beam intensity of the lost beam on the collimator and meanwhile, the Fig. 8(c) shows the beam intensity of the lost beam on the vacuum chamber. As a result of the limitation of the lattice and the positions of the collimators, some particles hit on the vacuum chamber wall and the average collimation efficiency can only reach $54.82 \%$. As mentioned above, the collimation efficiency can be optimized with different factors except the fixed longitudinal positions for the CSRm. In the following

TABLE III. Parameters of the prototype collimators.

$\left.\begin{array}{lc}\hline \hline \text { Material } & \begin{array}{c}\text { Copper } \\ \text { Coating }\end{array} \\ \text { Wow desorption } \\ \text { rate material }\end{array}\right)$




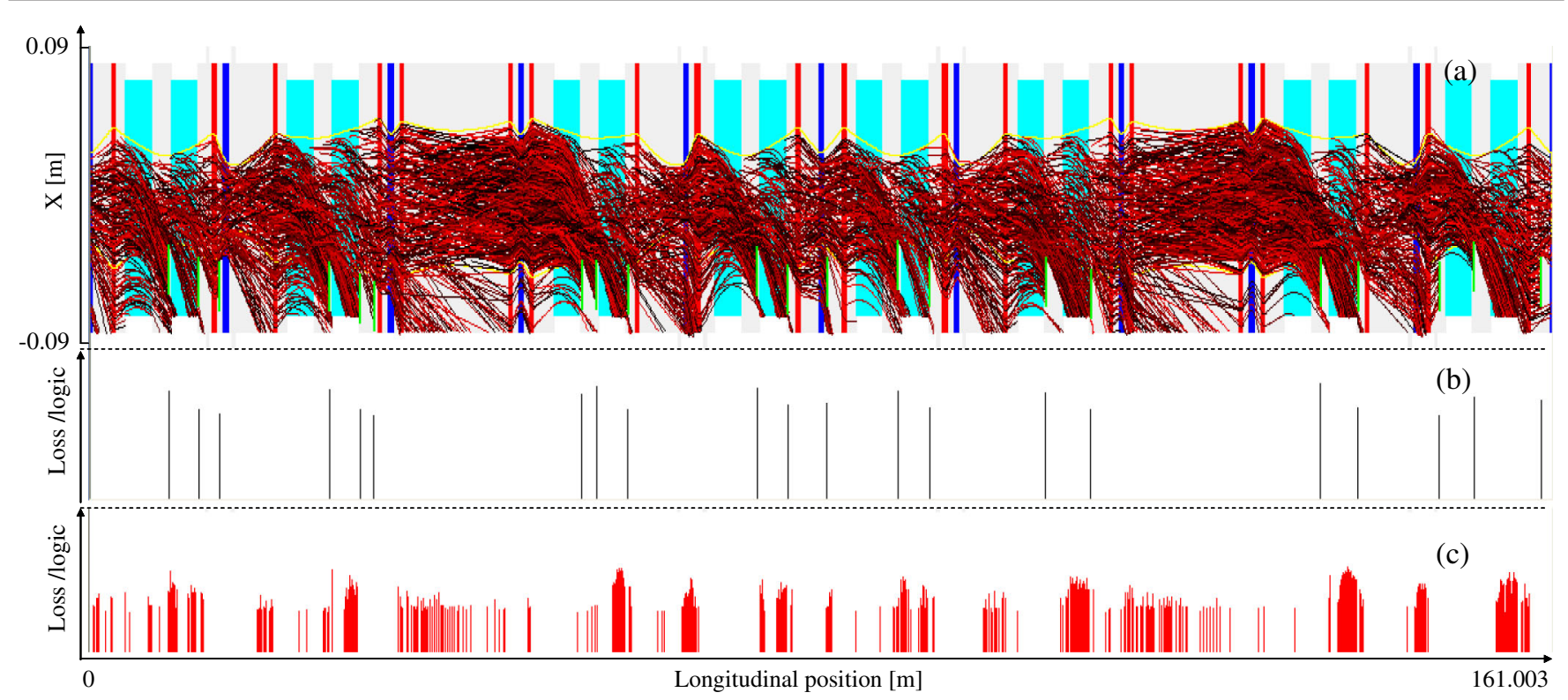

FIG. 8. Simulation results for one electron-loss ${ }^{238} \mathrm{U}^{33+}$ : (a) Beam loss trajectory. (b) Beam losses on the collimators. (c) Beam losses on the vacuum chamber. Loss/logic means: the logarithmic value of the lost beam intensity at each position.

sections, only the one electron-loss charge exchange process for ${ }^{238} \mathrm{U}^{32+}$ is simulated to optimize the collimation efficiency as an example. The investigation for electroncapture processes is not listed here, in the respect that it is carried out with the same procedure as the above process. In the future measurement, two rectangular block collimators will be installed at both sides of the vacuum chamber to catch electron-capture and electron-loss particles.

\section{A. Collimator length in the horizontal direction}

When the aperture of the vacuum chamber is large and the dimension of the collimator is not optimized, some lost particles will pass across the collimator and hit on the vacuum chamber. Meanwhile, the collimator cannot be too long because of the higher cost of the material and the difficulty to move it precisely. According to the beam trajectory at the collimator positions, the lengths of the collimators are optimized. The calculation of collimation efficiency is carried out 20 times with Gaussian distribution for charge exchanged particles in the phase space. The average value is obtained from 20 samples. The maximum and minimum deviations of the collimation efficiency are viewed as the error bars. The collimation efficiency evolution with collimator variable length for one electron-loss process is shown in Fig. 9. From the result, the collimator length in the horizontal direction has effects on the collimation efficiency. In order to save the budget and control the collimator precisely, the length of 17 collimators is $40 \mathrm{~mm}$ and that of the others is $50 \mathrm{~mm}$ in the horizontal direction. The collimation efficiency can reach $60.3 \%$ under this condition. The locations and lengths of the collimators are the basis for the next simulation.

\section{B. Collimator distance to the beam edge}

The most important rule for installing the collimator is that the collimator cannot decrease the transverse acceptance of the machine and meanwhile, it should catch the lost particles as much as possible. The collimation efficiency reaches maximum when the collimators are located at the edge of the beam envelope when there is no beam orbit distortion. The beam edge is defined by the beam envelope with $5 \sigma$ emittance. However, during the machine operation, the beam orbit will be distorted due to the alignment and field errors of the magnets. When the collimator that is located at the beam envelope edge refers to the ideal central orbit, the circulating accumulated

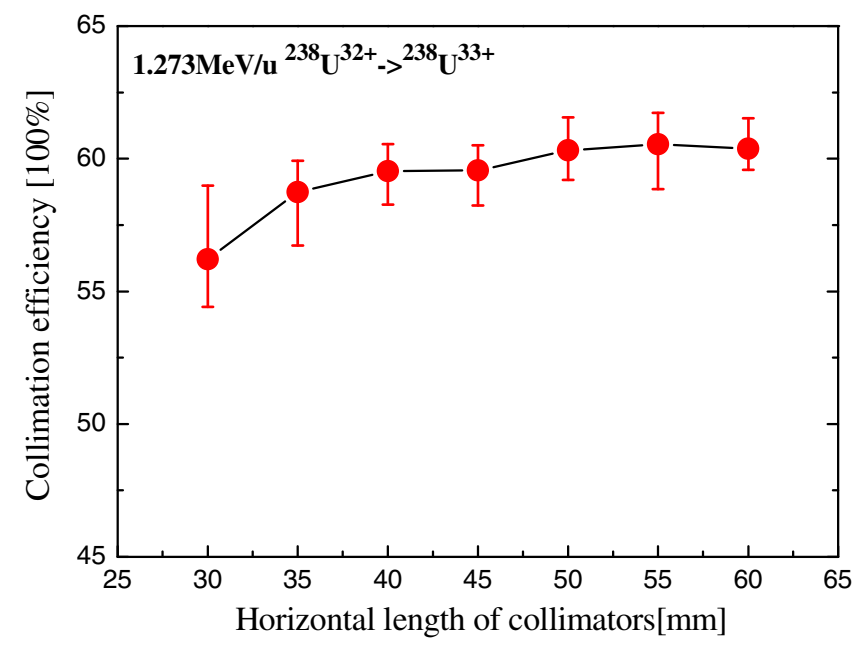

FIG. 9. Collimation efficiency with variable horizontal length of the collimators. 


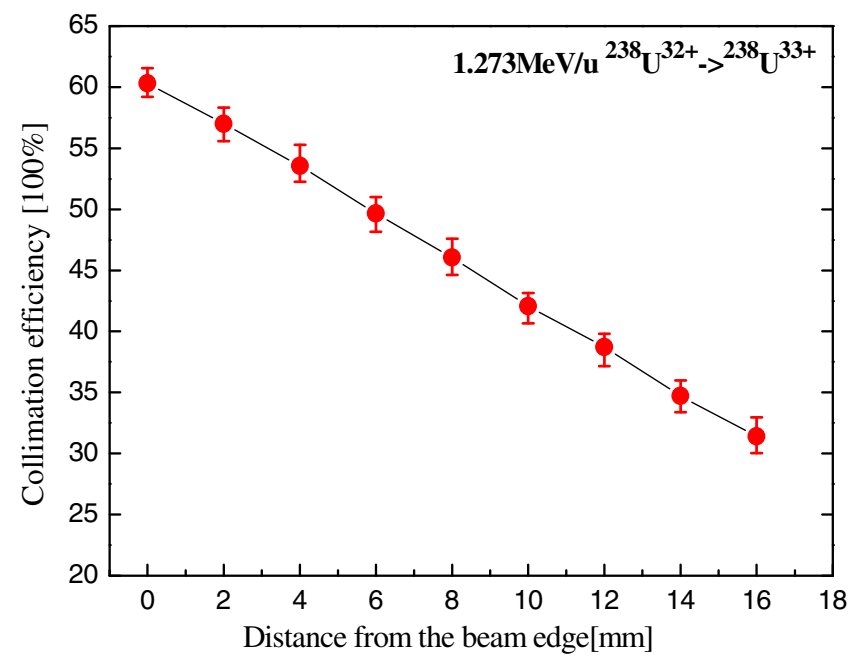

FIG. 10. Collimation efficiency with variable collimators transverse position.

normal beam is easily hit on the collimators because of the beam orbit distortion. Based on the fixed locations and lengths of the collimators, the collimation efficiency is simulated by adjusting the distance from the collimator to the beam edge at a certain beam emittance. The calculation result is illustrated in Fig. 10 where the collimation efficiency decreases quickly when the collimators are pulled out from the beam edge. Therefore the collimator positions will be adjusted according to the actual beam orbit in order to get much higher collimation efficiency when they are used in the CSRm.

\section{Beam emittance}

In the CSRm, a multiturn injection method with electron cooling is adopted to accumulate the heavy ion beams. A closed orbit bump of variable amplitude is provided that allows injecting the beam at an increasing distance from the ideal orbit and thus filling gradually the machine acceptance $(150 \pi \mathrm{mm} \mathrm{mrad})$. Then the beam will be cooled down to leave the extra phase space for the next multiturn injection. During the electron cooling process, the beam emittance will be decreased from the machine acceptance to $35 \pi \mathrm{mm} \mathrm{mrad}$ in the horizontal phase space [23]. The collimation efficiency for one electron-loss process is simulated with the evolution of the horizontal beam emittance is showed in Fig. 11. The result shows that lower beam emittance that is cooled down by the electron cooling contributes to the higher catchability of the charge exchanged particles. Furthermore, collimator movement space in a horizontal direction increases when the beam emittance gradually becomes lower. This horizontal movement space increase reduces the possibility that collision happens between the normal circulating beams and the collimators.

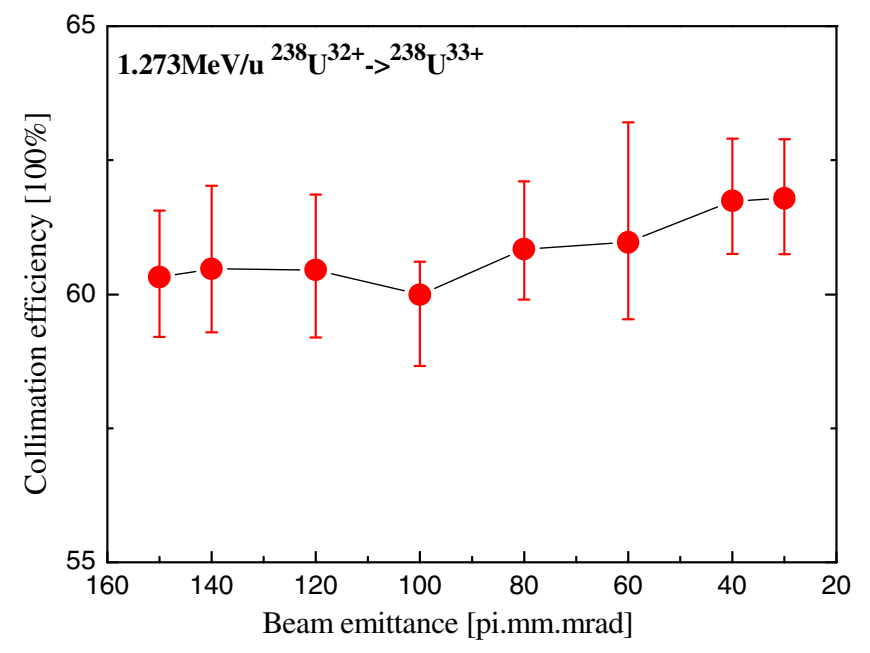

FIG. 11. Collimation efficiency with beam emittance.

\section{Beam distribution}

In this simulation software package, two types of distributions, $2 \sigma$ cutoff Gaussian distribution and rectangular uniform distribution are adopted to simulate the particle motion. The collimation efficiency for rectangular uniform distribution is also calculated to make the comparison with the Gaussian distribution. There are no significant differences between these two distributions for the CSRm. Actually, the collimation efficiency for Gaussian distribution should be higher than the rectangular uniform distribution when the accelerator lattice is optimized [24]. The collimation efficiency for one electron-loss process with two types of different distributions is shown in Fig. 12. All the simulation results in this paper are based on the Gaussian distribution, which is commonly used to evaluate the beam properties and is closer to the real measured distribution in the CSRm [25].

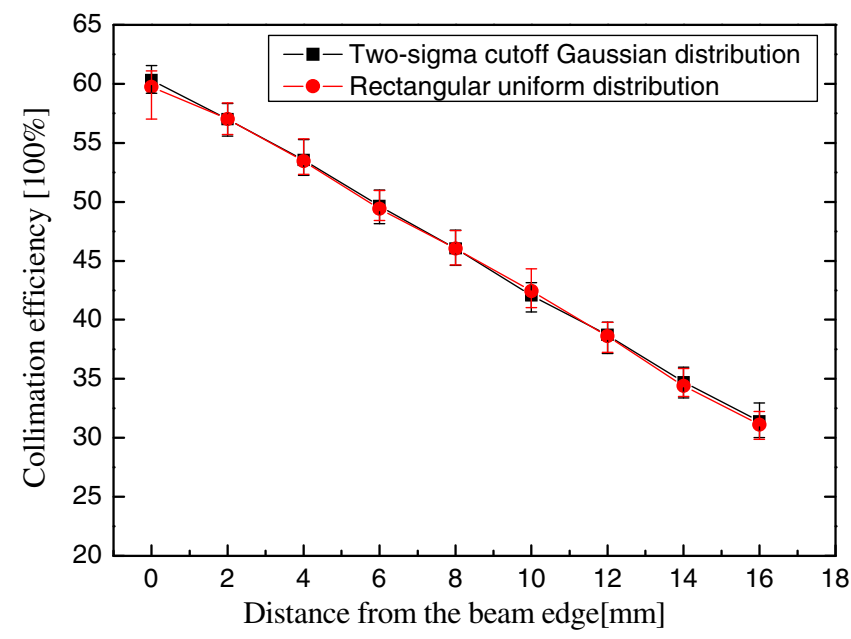

FIG. 12. Collimation efficiency with distance from collimator to the beam edge under two types of distributions. 


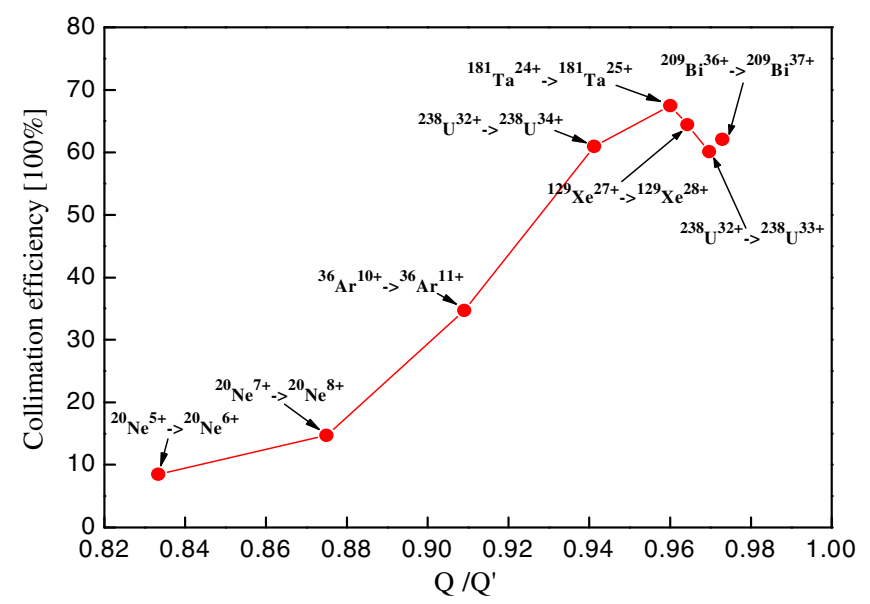

FIG. 13. Collimation efficiency of different ions. The $Q$ is the nominal ion charge state and the $Q^{\prime}$ is the charge state of the ion after charge exchange process.

\section{E. Collimation efficiency of different ions}

CSRm can accelerate the heavy ion beams from proton to uranium. Many other intermediate charge state heavy ion beams have been accelerated in the CSRm such as ${ }^{78} \mathrm{Kr}^{28+}$, ${ }^{129} \mathrm{Xe}^{27+},{ }^{209} \mathrm{Bi}^{36+}$, and so on [26]. Those intermediate charged beams also can capture or lose electrons due to the collision with the residual gas. The collimation efficiency of different intermediate charge state ion beams in the CSRm for an electron-loss process is illustrated in Fig. 13 with all optimum parameters mentioned above. Because there is no free space for the collimator motion in the longitudinal beam direction, the collimation efficiency for these heavy ions cannot be optimized again. In the future, in order to improve collimation efficiency for different ions, longitudinal movable collimators, for instance, will be designed in the new facility (HIAF). During the machine operation, intermediate charge state particles of higher collimation efficiency will be accumulated for the physics experiment.

\section{LINEAR ELEMENT ERRORS}

It is well known that the design of an accelerator differs from the operating conditions because of the idealized character of the forces used in the design stages [27]. Many errors, such as misalignment of the linear elements, magnetic field errors, and so on will lead to a distortion of the beam orbit and the changes of the lattice functions. The trajectories of the lost particles are also affected by the orbit distortion and then cause the lower collimation efficiency. Thus it is desirable to get an estimation of the effects of errors on collimation efficiency of an accelerator. These effects in turn dictate the tolerances on these errors. In this paper, only the linear dynamic particle motion is simulated. All the nonlinear effects like the kinematic effect, magnetic fringe field, and high order multiple errors are ignored. The radius of curvature is assumed piecewise constant and there is no energy change of the particle. Because the sextupoles and skew quadrupoles are viewed as the drift in this simulation, the coupled motion caused by those elements and all nonlinear contributions to the equations of motion are neglected. In this paper, only the linear coupling produced by the quadrupole rotation errors is taken into consideration.

\section{A. Linearized equation in the presence of errors}

For a particle in the accelerator, the linearized betatron equation of particle motion governed by Hill's equation is given by

$$
u^{\prime \prime}+h_{u} K_{u} u=0
$$

where $u$ represents either horizontal or vertical coordinate, $K_{u}$ is the effective focusing function, and $h_{u}$ is a sign to represent the horizontal or vertical focusing. Because accelerator components usually have uniform or nearly uniform magnetic fields, the focusing functions $K_{u}$ are piecewise constant [15]. A new driving item in this differential equation will affect the beam trajectory when there is an error $\Delta$ and then the particle motion equation becomes [27,28]

$$
u^{\prime \prime}+h_{u} K_{u} u=\Delta f_{u}(z),
$$

where $f_{u}(z)$ is the force that related to the error. The linear two-order homogeneous differential equation (4) can be solved and then the linear transfer matrix of the particle motion is given by

$$
\left(\begin{array}{c}
u_{n} \\
u_{n}^{\prime}
\end{array}\right)=\left(\begin{array}{ll}
R_{u n 11} & R_{u n 12} \\
R_{u n 21} & R_{u n 22}
\end{array}\right) *\left(\begin{array}{c}
u_{n 0} \\
u_{n 0}^{\prime}
\end{array}\right) .
$$

The inhomogeneous differential equation (5) for the perturbed betatron oscillation can be solved by the extended $3 \times 3$ transfer matrix which is expressed as

$$
\left(\begin{array}{c}
u_{n} \\
u_{n}^{\prime} \\
\Delta_{n}
\end{array}\right)=\left(\begin{array}{ccc}
R_{u n 11} & R_{\text {un } 12} & R_{\text {un } 13} \\
R_{\text {un } 21} & R_{\text {un } 22} & R_{\text {un } 23} \\
0 & 0 & 1
\end{array}\right) *\left(\begin{array}{c}
u_{n 0} \\
u_{n 0}^{\prime} \\
\Delta_{n}
\end{array}\right) .
$$

If the matrix items $R_{u n 13}$ and $R_{u n 23}$ can be obtained, then the charge exchanged particles' trajectory resulting from different types of errors can be analyzed. In the following sections, different extended transfer matrices related to the element errors will be investigated by this method.

\section{B. Quadrupole errors}

\section{Transverse position errors of quadrupole}

The transverse position errors of the quadrupole will produce transverse kicks and consequently a direct distortion of the beam orbit. Because a horizontally focusing 
quadrupole is a vertically defocusing quadrupole as well, only the process for driving the matrix for a horizontal focusing quadrupole is introduced. The horizontal and vertical transfer matrix with transverse position errors of the quadrupole is given in Appendix A.

The transverse misplacements of quadrupoles are the most important factors that cause the orbit distortion in the accelerator, and they will affect the collimation efficiency accordingly. In this simulation, a random value that represents the transverse position errors is generated and then applied to each quadrupole; meanwhile both the horizontal and the vertical errors are set to the same value. The simulation result that is performed with random Gaussian distribution is illustrated in Fig. 14(a). The error bar shows maximum and minimum deviation of the collimation efficiency of 20 samples with various transverse position errors of quadrupole magnets. The error bar increases with the larger random quadrupole transverse position errors. From this result, the transverse alignment of the quadrupole must be controlled less than $0.7 \mathrm{~mm}(1 \sigma)$ from the ideal path to maintain the optimum collimation efficiency.

\section{Quadrupole magnetic field errors}

Quadrupole fields determine the betatron function as well as the phase advance or tune in a circular accelerator. The magnetic field quality is a result of the mechanical design of the magnet and the stability of the power supply providing the electrical current. For a synchrotron, quadrupole field error can cause betatron tune oscillation and emittance increase. In this paper, the extended transfer matrix for a horizontal focusing quadrupole with magnetic field errors is also investigated and can be found in Appendix B.

According to the extended transfer matrix, the particle trajectory changed due to the quadrupole magnetic field errors depend on not only the quadrupole strength and errors, but also the particle positions. Different field deviations will impose on the particles according to their location in the phase space. This section only addresses the effect of quadrupole field errors that arise from the quadrupole power supply ripple. The effect of magnet-to-magnet variation due to the magnet itself is not included. Applying the extended transfer matrix, the collimation efficiency of the CSRm for uranium ${ }^{238} \mathrm{U}^{32+}$ for one electron-loss process is simulated. The simulation result is illustrated in Fig. 14(b). In the practice of accelerator operation, the collimation efficiency will not be affected seriously when the ripple of quadrupole power supply is better than $10^{-5}$.

\section{Quadrupole rotation errors around the longitudinal axis}

The quadrupole can be rotated by a small angle with respect to the reference coordinate system owing to the mechanical installation errors. Quadrupole rotations create linear coupling between the two transverse directions, $x$ (horizontal) and $y$ (vertical), in the equations of particle motion. The quadrupole rotation diagram is illustrated in detail in Ref. [29]. The transfer matrix for quadrupole with rotation errors can be found in Appendix C. As mentioned above, skew quadrupoles that are used to correct a linear coupling effect is not considered in this simulation.

According to the extended transfer matrix, the collimation efficiency of the CSRm for uranium ${ }^{238} \mathrm{U}^{32+}$ for one electron-loss process is investigated with the quadrupole rotation errors. The calculation result is illustrated in Fig. 14(c). This simulation result shows that quadrupole rotation errors have few influences on the collimation efficiency. Actually, the effect of such an error can be neglected when the rotation angle is smaller than $0.5 \mathrm{mrad}(1 \sigma)$.

\section{Other quadrupole errors}

There are also some deviations of the quadrupole length due to the mechanical fabrication errors. Because of the relationship $\frac{\Delta K^{\prime}}{K^{\prime}}=\frac{\Delta L}{L}$, the extended transfer matrix for the quadrupole length errors is the same as the quadrupole magnetic field errors. The method to calculate the collimation efficiency with quadrupole magnet length errors in
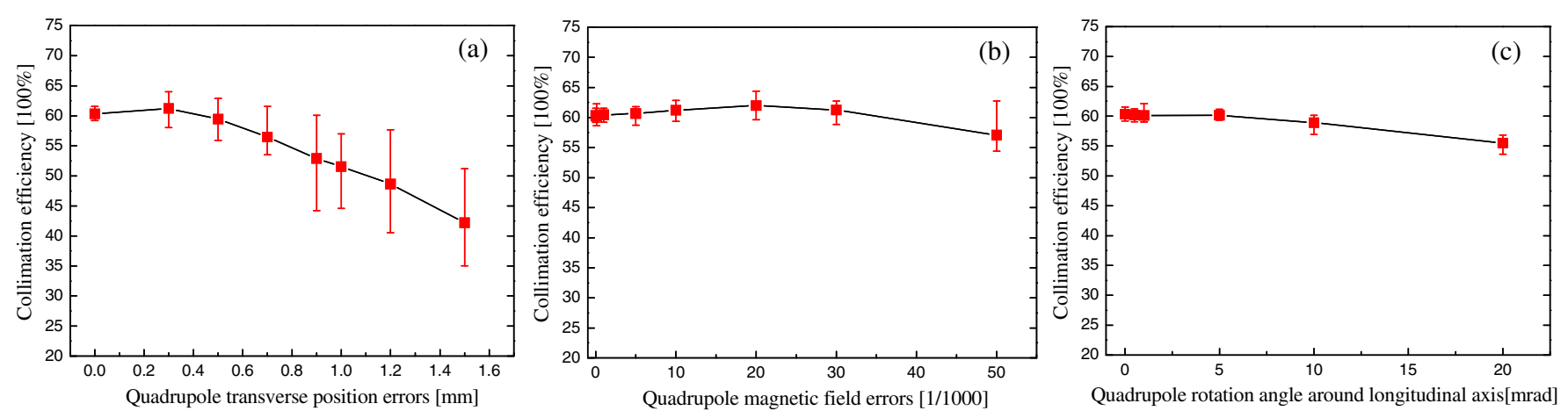

FIG. 14. Collimation efficiency with (a) quadrupole transverse position errors and (b) quadrupole magnetic field errors. The unit of $x$ axis $[1 / 1000]$ is the ratio of the magnetic gradient deviation to the theoretical setting value of the quadrupole at a certain magnetic gradient. (c) Quadrupole rotation errors around the longitudinal axis. 
the CSRm is similar to the one for quadrupole magnetic field errors.

During the accelerator operation, there are also some other types of quadrupole errors, such as the pitch of the quadrupole magnet in the transverse dimension and the quadrupole motion in the longitudinal direction. However, those types of errors are neglected in this paper owing to their small influences on the collimation efficiency.

\section{Dipole errors}

In the synchrotron, a bending dipole is used to provide the magnetic force to guide the particles circulating in a closed orbit. Dipole errors can cause an angular deflection of the beam, which thus leads to the beam orbit oscillation around the design central orbit. The charge exchanged beam is easily lost in the vertical direction as a result of the beam orbit distortion and the smaller vertical vacuum chamber. In this section, the collimation efficiency evolution caused by the dipole errors is investigated in the same way as the quadrupole errors.

\section{Transverse position errors of dipole}

The particle motion equation in the dipole that is expressed by Eq. (4) is the same as the quadrupole only that the focusing factor is as $K_{x}=\frac{1-n_{x}}{\rho^{2}}$, and $K_{y}=\frac{n_{x}}{\rho^{2}}$. Here, the $\rho$ is the bending radius and the $n_{x}$ is the gradient factor in the dipole. In this paper, the gradient factor is neglected and then the focusing factor for particles' motion in a dipole becomes $K_{x}=\frac{1}{\rho^{2}}$, and $K_{y}=0$. Because of the same motion equation for the particle in the dipole and quadrupole and the same forces related to the transverse position errors, the extended transfer matrix for transverse position errors is transformed according to the new focusing factor in the horizontal direction given directly in Appendix D.

As it is known that $K_{y}=0$, the dipole vertical position errors only affect the effective aperture rather than the beam trajectory. The simulation result of collimation efficiency for uranium ${ }^{238} \mathrm{U}^{32+}$ for one electron loss process with dipole transverse position errors in the CSRm is shown in Fig. 15(a). In the CSRm, the dipole transverse position with respect to the ideal beam path is controlled less than $0.5 \mathrm{~mm}$ $(1 \sigma)$ and hence it will not lead to a rapid decrease of the collimation efficiency according to the simulation result.

\section{Dipole magnetic field errors}

In general, dipole magnetic field errors are generated by a power supply ripple, ground vibration, mechanical vibration, and so on. Such an error is treated as a kicker in the lattice, which can lead to the orbit distortion and emittance growth. With Eq. (4) and the focusing factor for the particle motion in the dipole, the force that is related to the dipole magnetic field errors is $f_{x}(z)=-1 / \rho$, and $f_{y}(z)=0$. The extended transfer matrix is approximated and can be found in Appendix D.

The dipole magnetic field sometimes is modulated at a certain frequency. If the modulation frequency is equal to the betatron or synchrotron frequency, particle motion will be strongly perturbed. A modulation function with kick angle and angular frequency can replace the item $R_{x 23}$ to simulate the orbit oscillation. In this paper, this work is neglected and will be investigated for future research.

The collimation efficiency of the CSRm for uranium ${ }^{238} \mathrm{U}^{32+}$ with dipole magnetic field errors for the one electron-loss process is simulated and shown in Fig. 15(b). In this simulation, the dipole magnetic field errors that are affected by the power supply ripple are considered. The magnetic field error that is arising from the magnet manufacturer is neglected. When the dipole magnetic field deviates $10 \%$ from the design parameter, the collimation efficiency increases because more particles are lost on the collimators. However, the beam orbit of reference ions ${ }^{238} \mathrm{U}^{32+}$ will be distorted and then the normal ions ${ }^{238} \mathrm{U}^{32+}$ will be lost on the collimators. The dipole power supply ripple is better than $5 \times 10^{-5}$ in practice and then has little influence on the collimation efficiency.
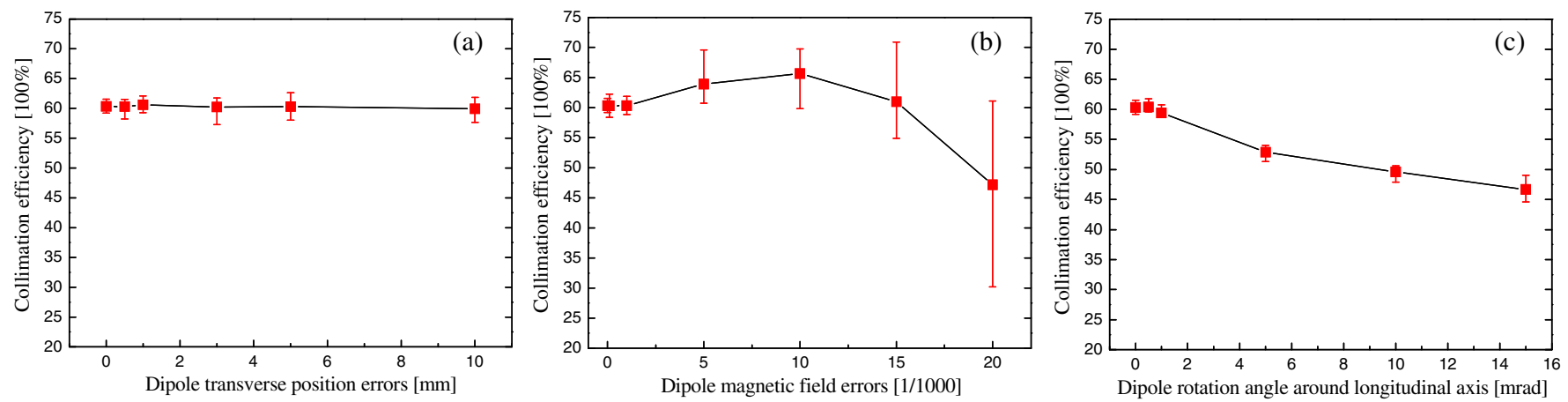

FIG. 15. Collimation efficiency with (a) dipole transverse position errors and (b) dipole magnetic field errors. The unit of $x$ axis $[1 / 1000]$ is the ratio of the magnetic field deviation to the theoretical setting value of the dipole at a certain magnetic field. (c) Dipole rotation errors around the longitudinal axis. 


\section{Dipole rotation errors around longitudinal axis}

Rotation around the three coordinate axes of the dipole also possibly exist due to installation errors in reality. The beam circulating around the accelerator will receive an unwanted vertical kick when a dipole is rotated about the magnet axis. The transverse dipole rotation around the design central orbit can be neglected because of the small impact on the beam orbit and the collimation efficiency. Coordinates of the particles are changed arising from the rotation in the longitudinal direction. With the approximation, the force related to the dipole rotation errors is $f_{x}(z)=0$, and $f_{y}(z)=-1 / \rho$, then the vertical extended transfer matrix is approximated and can be found in Appendix D.

Some charge exchanged particles are lost on the vertical vacuum chamber when the vertical beam orbit distortion is caused by the dipole rotation errors. The collimators are able to catch certain lost particles with $30 \mathrm{~mm}$ length in the vertical direction. The collimation efficiency with this type of error in the CSRm for uranium ${ }^{238} \mathrm{U}^{32+}$ for one electronloss process is illustrated in Fig. 15(c). From the simulation result, the collimation efficiency will be maintained at more than $55 \%$ when the rotation angle is less than $1 \mathrm{mrad}(1 \sigma)$. Meanwhile, the alignment result of the dipole rotation is better than $0.5 \mathrm{mrad}(1 \sigma)$ in the real machine operation.

\section{Other types of dipole error}

There are also some deviations of the dipole length generated by the magnet manufacturer's precision accuracy. As with the quadrupole length errors, the extended matrix for this case is the same as the dipole magnetic field errors with the relationship $\frac{\Delta B}{B}=-\frac{\Delta L}{L}$. So the investigation on the collimation efficiency with the dipole length errors will not be carried out again.

Other types of errors for the dipole such as rotation in the transverse direction will not be addressed in this paper because of their small perturbation on the beam trajectory.

\section{ACTUAL ALIGNMENT ERRORS}

According to the calculation results mentioned above, the distortion of the beam orbit and the growth of the betatron oscillation amplitude will result in lower collimation efficiency.

In order to simulate the effect of quadrupole misalignment and dipole rotation errors on the collimation efficiency in the CSRm, actual machine parameters are adopted. Figures 16 and 17 show the transverse position errors of the quadrupoles before the mechanical adjustment along the CSRm and the rotation errors of 16 dipoles along the CSRm, respectively [30]. Quadrupole and dipole magnetic field errors are both set as $5 \times 10^{-5}$ with $3 \sigma$ Gaussian distribution for each optical element. Quadrupole rotation errors and dipole transverse position errors are neglected due to their limited effect.

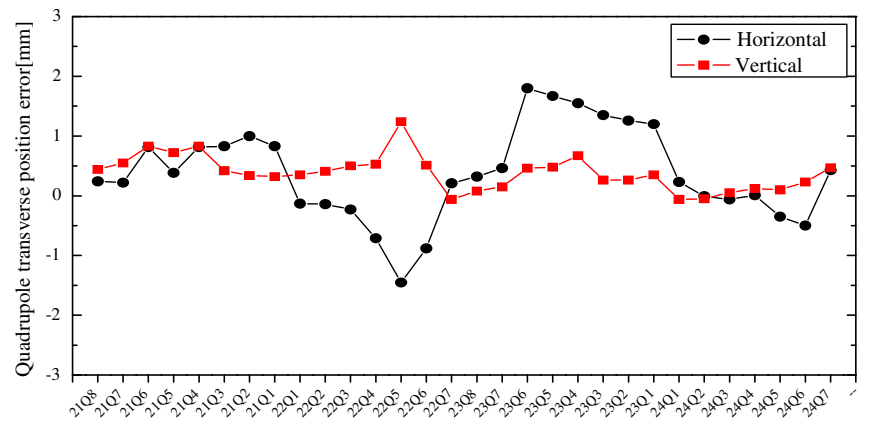

FIG. 16. Quadrupole transverse position error.

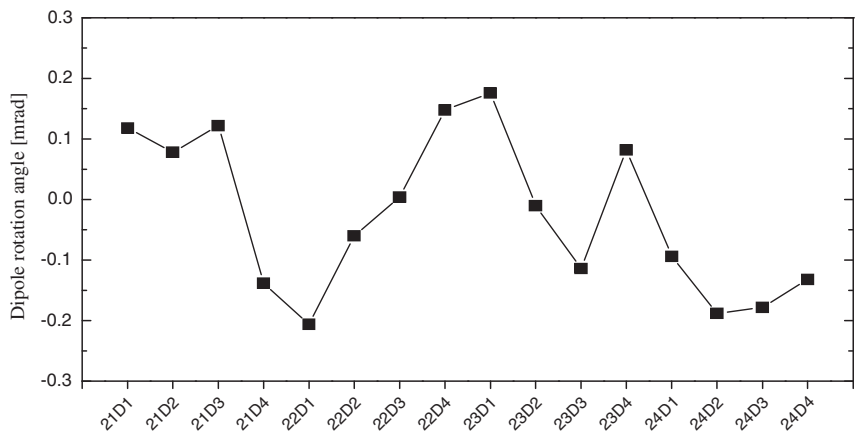

FIG. 17. Sixteen dipoles' rotation angle around the longitudinal axis.

The maximum vertical closed orbit of the normal beam ${ }^{238} \mathrm{U}^{32+}$ is $9 \mathrm{~mm}$ and $2 \mathrm{~mm}$ in the horizontal dimension. Based on the actual misalignment parameters and power supply ripple, the average collimation efficiency is calculated as $53.08 \%$. The deviation range is $[1.56 \%, 1.25 \%]$. Colleagues from the alignment group will adjust the magnet installation precision to fulfill the design requirements of the CSRm $[1,30]$.

According to the simulation result, good restrictions of the alignment errors contribute to a higher collimation efficiency of charged exchanged heavy ion particles. Meanwhile, reasonable restrictions of all errors can prevent the interaction between the normal circulating beam and the collimators. The machine acceptance cannot be decreased by the beam orbit distortion and collimator movement.

\section{CONCLUSIONS}

In this paper, the beam losses driven by particle ionization process are simulated in the CSRm for the first time. A collimation system is designed to catch the charge exchanged particles as much as possible. The collimation efficiency is optimized under different collimator designs and simulated with various dipole and quadrupole field errors. Finally, the collimation efficiency is also calculated based on the actual machine parameters. The conclusion is drawn as follows: 1 . Based on the limit installation space of the CSRm, only 21 collimators can be installed in this circular accelerator. The lengths of collimators are designed 
to be $50 \mathrm{~mm}$ and $40 \mathrm{~mm}$. According to the calculation result, lower emittance will contribute to a higher efficiency. There is no significant difference between $2 \sigma$ cutoff Gaussian distribution and rectangular uniform distribution. The highest efficiency for uranium ${ }^{238} \mathrm{U}^{32+}$ for one electronloss process is about $60 \%$. 2 . The collimation efficiency will be affected by the element alignment and magnetic field errors. Simulation results illustrate that the most important factor that results in the beam orbit distortion and collimation efficiency decrease is the quadrupole transverse misalignments. Other effects, such as magnetic field errors, magnet rotation errors, and magnet length errors can be neglected. In general, the misplacements of magnets with respect to the ideal beam path must be controlled and horizontal positions of the collimators must be adjusted in accordance with the circulating beam closed orbit. 3. As mentioned above, the collimation efficiency is approximate $60 \%$. Therefore, only two prototype collimators will be tested and installed in the space between two dipoles first. Two prototype collimators will be manufactured with copper coated by gold and nickel. The mechanical design of these two collimators has been finished. It will be installed and tested in the CSRm at the beginning of 2015.

Further simulation including the nonlinear and collective effects such as space charge and dynamic vacuum desorption will be carried out for the next step. The collimation system will be considered and installed in the CSRm to control the beam losses finally.

\section{ACKNOWLEDGMENTS}

We thank Dr. Peter Spiller (GSI) for providing P. L. an opportunity to work at GSI to learn the collimation. We also thank Dr. Lars Bozyk (GSI) and Dr. Carsten Omet (GSI) for giving P. L. much help on the beam simulation code. Special thanks to Dr. Lars Bozyk for his directions on collimator design and help during the prototype test experiment. This work is supported by the National Natural Science Foundation of China (Project No. 11305227).

\section{APPENDIX A: EXTENED TRANSFER MATRIX FOR QUADRUPOLE TRANSVERSE POSITION ERRORS}

Suppose a quadrupole is displaced horizontally with respect to the beam axis by a small amount $\Delta x$, then the ideal particle position becomes

$$
x_{t}=x-\Delta x .
$$

Then the linearized betatron equation of particle motion Eq. (4) in the horizontal direction is written as

$$
(x-\Delta x)^{\prime \prime}+K_{x}(x-\Delta x)=0 .
$$

Having been transformed from Eq. (A2), the particle motion equation with alignment errors in the horizontal direction is then given by

$$
x^{\prime \prime}+K_{x} x=\Delta x \cdot K_{x} .
$$

The force related to the alignment error is $f_{x}(z)=K_{x}$. The linear two-dimensional transfer matrix for the focusing quadrupole in the horizontal direction is $[15,28]$

$$
\left(\begin{array}{cc}
\cos (\sqrt{K} L) & \frac{1}{\sqrt{K}} \sin (\sqrt{K} L) \\
-\sqrt{K} \sin (\sqrt{K} L) & \cos (\sqrt{K} L)
\end{array}\right) .
$$

For the nonhomogenous differential equation (A3), the matrix item $R_{x 13}$ can be calculated by [28]

$$
\begin{aligned}
R_{x 13}= & -\frac{\cos \left(\sqrt{K_{x}} z\right)}{\sqrt{K_{x}}} \int_{z_{0}}^{z} f(\theta) \sin \left(\sqrt{K_{x}} \theta\right) d \theta \\
& +\frac{\sin \left(\sqrt{K_{x}} z\right)}{\sqrt{K_{x}}} \int_{z_{0}}^{z} f(\theta) \cos \left(\sqrt{K_{x}} \theta\right) d \theta,
\end{aligned}
$$

where the length of the quadrupole is $L=z-z_{0}$. According to the relationship $R_{x 23}=R_{x 13}^{\prime}$, the extended transfer matrix for a horizontal focusing quadrupole with alignment error is

$$
\left(\begin{array}{ccc}
\cos \left(\sqrt{K_{x}} L\right) & \frac{1}{\sqrt{K_{x}}} \sin \left(\sqrt{K_{x}} L\right) & 1-\cos \left(\sqrt{K_{x}} L\right) \\
-\sqrt{K_{x}} \sin \left(\sqrt{K_{x}} L\right) & \cos \left(\sqrt{K_{x}} L\right) & \sqrt{K_{x}} \sin \left(\sqrt{K_{x}} L\right) \\
0 & 0 & 1
\end{array}\right) .
$$

By solving the focusing quadrupole motion equation in the vertical direction, the extended transfer matrix for this case is

$$
\left(\begin{array}{ccc}
\operatorname{ch}\left(\sqrt{K_{y}} L\right) & \frac{1}{\sqrt{K_{y}}} \operatorname{sh}\left(\sqrt{K_{y}} L\right) & 1-\operatorname{ch}\left(\sqrt{K_{y}} L\right) \\
\sqrt{K_{y}} \operatorname{sh}\left(\sqrt{K_{y}} L\right) & \operatorname{ch}\left(\sqrt{K_{y}} L\right) & -\sqrt{K_{y}} \operatorname{sh}\left(\sqrt{K_{y}} L\right) \\
0 & 0 & 1
\end{array}\right) .
$$

In the thin-lens approximation, only the particle angle will be changed due to the errors; the item $R_{x 13}$ will then become zero, $R_{x 23}$ is $K L$ in the horizontal direction, $R_{y 13}$ will become zero, and $R_{y 23}$ is $-K L$ in the vertical direction. The famous accelerator simulation code Winagile [14] adopts this thin-lens approximation in the algorithm.

\section{APPENDIX B: EXTENED TRANSFER MATRIX FOR QUADRUPOLE MAGNETIC FIELD ERRORS}

Suppose there is the quadrupole magnetic perturbing field $\Delta B^{\prime}$ that refers to the ideal field $B^{\prime}$, then the focusing function $K_{x}$ becomes $K_{x}\left(1+\frac{\Delta B^{\prime}}{B^{\prime}}\right)$. The particle motion Eq. (4) can be rewritten as [28] 


$$
x^{\prime \prime}+K_{x} x=-\frac{\Delta B^{\prime}}{B^{\prime}} K_{x} x
$$

where the force associated with the magnetic field error is $f_{x}(z)=-K_{x} x$. By using Eq. (A5) and taking approximate calculation, the matrix item $R_{x 13}$ can be calculated as $-x\left[1-\cos \left(\sqrt{K_{x}} L\right)\right]$, and $R_{x 23}$ as $-x\left[\sqrt{K_{x}} \sin \left(\sqrt{K_{x}} L\right)\right]$. With quadrupole magnetic field errors, the extended transfer matrix in the horizontal direction is

$$
\left(\begin{array}{ccc}
\cos \left(\sqrt{K_{x}} L\right) & \frac{1}{\sqrt{K_{x}}} \sin \left(\sqrt{K_{x}} L\right) & -x\left[1-\cos \left(\sqrt{K_{x}} L\right)\right] \\
-\sqrt{K_{x}} \sin \left(\sqrt{K_{x}} L\right) & \cos \left(\sqrt{K_{x}} L\right) & -x \sqrt{K_{x}} \sin \left(\sqrt{K_{x}} L\right) \\
0 & 0 & 1
\end{array}\right)
$$

and the extended transfer matrix in the vertical direction is

$$
\left(\begin{array}{ccc}
\operatorname{ch}\left(\sqrt{K_{y}} L\right) & \frac{1}{\sqrt{K_{y}}} \operatorname{sh}\left(\sqrt{K_{y}} L\right) & -y\left[1-\operatorname{ch}\left(\sqrt{K_{y}} L\right)\right] \\
\sqrt{K_{y}} \operatorname{sh}\left(\sqrt{K_{y}} L\right) & \operatorname{ch}\left(\sqrt{K_{y}} L\right) & y \sqrt{K_{y}} \operatorname{sh}\left(\sqrt{K_{y}} L\right) \\
0 & 0 & 1
\end{array}\right) .
$$

\section{APPENDIX C: EXTENED TRANSFER MATRIX FOR QUADRUPOLE ROTATION ERRORS}

Suppose the rotation angle around the longitudinal direction is $\Delta \varphi$; the beam trajectory in the transverse direction can then be expressed by [28]

$$
\begin{aligned}
& x_{t}=x \cos (\Delta \varphi)+y \sin (\Delta \varphi), \\
& y_{t}=y \cos (\Delta \varphi)-x \sin (\Delta \varphi),
\end{aligned}
$$

where the $x_{t}$ and $y_{t}$ are the beam coordinates in the ideal coordinate system and $x$ and $y$ are the beam position in the new rotation coordinate system, respectively. When the rotation angle is small, Eq. (C1) can be approximated as

$$
\begin{aligned}
& x_{t}=x+y(\Delta \varphi), \\
& y_{t}=y-x(\Delta \varphi) .
\end{aligned}
$$

Using the Eq. (C2) and Eq. (4), the new particle motion equation can be derived as

$$
\begin{aligned}
x^{\prime \prime}+h_{x} K_{x} x & =-\left(y^{\prime \prime}+h_{x} K_{x} y\right)(\Delta \varphi), \\
y^{\prime \prime}+h_{y} K_{y} y & =-\left(x^{\prime \prime}+h_{y} K_{y} x\right)(\Delta \varphi) .
\end{aligned}
$$

Because the relationship $h_{x}=-h_{y}$ and the assumption $K_{x}=K_{y}$, the above equation can be expressed as

$$
\begin{aligned}
x^{\prime \prime}+K x & =-2 K y(\Delta \varphi), \\
y^{\prime \prime}+K y & =2 K x(\Delta \varphi),
\end{aligned}
$$

where the force associated with the quadrupole rotation error is $f_{x}(z)=-2 K y, \quad f_{y}(z)=-2 K x$. By using Eq. (A5), the extended transfer matrix for the quadrupole rotation errors is expressed as

$$
\left(\begin{array}{ccc}
\cos \left(\sqrt{K_{x}} L\right) & \frac{1}{\sqrt{K_{x}}} \sin \left(\sqrt{K_{x}} L\right) & -2 y\left[1-\cos \left(\sqrt{K_{x}} L\right)\right] \\
-\sqrt{K_{x}} \sin \left(\sqrt{K_{x}} L\right) & \cos \left(\sqrt{K_{x}} L\right) & -2 y \sqrt{K_{x}} \sin \left(\sqrt{K_{x}} L\right) \\
0 & 0 & 1
\end{array}\right),
$$

and the extended transfer matrix in the vertical direction is

$$
\left(\begin{array}{ccc}
\operatorname{ch}\left(\sqrt{K_{y}} L\right) & \frac{1}{\sqrt{K_{y}}} \operatorname{sh}\left(\sqrt{K_{y}} L\right) & -2 x\left[1-\operatorname{ch}\left(\sqrt{K_{y}} L\right)\right] \\
\sqrt{K_{y}} \operatorname{sh}\left(\sqrt{K_{y}} L\right) & \operatorname{ch}\left(\sqrt{K_{y}} L\right) & 2 x \sqrt{K_{y}} \operatorname{sh}\left(\sqrt{K_{y}} L\right) \\
0 & 0 & 1
\end{array}\right) .
$$




\section{APPENDIX D: EXTENED TRANSFER MATRIX FOR DIPOLE ERRORS}

1. Extend transfer matrix for dipole transverse position errors:

$$
\left(\begin{array}{ccc}
\cos \left(\frac{L}{\rho}\right) & \rho \sin \left(\frac{L}{\rho}\right) & 1-\cos \left(\frac{L}{\rho}\right) \\
-\frac{1}{\rho} \sin \left(\frac{L}{\rho}\right) & \cos \left(\frac{L}{\rho}\right) & \frac{1}{\rho} \sin \left(\frac{L}{\rho}\right) \\
0 & 0 & 1
\end{array}\right) .
$$

2. Extended transfer matrix for dipole magnetic field errors:

$$
\left(\begin{array}{ccc}
\cos \left(\frac{L}{\rho}\right) & \rho \sin \left(\frac{L}{\rho}\right) & -\rho\left[1-\cos \left(\frac{L}{\rho}\right)\right] \\
-\frac{1}{\rho} \sin \left(\frac{L}{\rho}\right) & \cos \left(\frac{L}{\rho}\right) & -\sin \left(\frac{L}{\rho}\right) \\
0 & 0 & 1
\end{array}\right)
$$

3. Extended transfer matrix for dipole rotation errors around longitudinal axis:

$$
\left(\begin{array}{ccc}
1 & L & 0 \\
0 & 1 & -\frac{L}{\rho} \\
0 & 0 & 1
\end{array}\right) .
$$

[1] J. W. Xia et al., Nucl. Instrum. Methods Phys. Res., Sect. A 488, 11 (2002).

[2] P. Li, Y. J. Yuan, J. C. Yang, J. W. Xia, W. Zhang, R. S. Mao, D. Y. Yin, and W. P. Chai, Nucl. Instrum. Methods Phys. Res., Sect. A 697, 17 (2013).

[3] Y. J. Yuan et al., HIRFL-CSR Annual Report, 2011 [http://english.imp.cas.cn/sp/ap/2011nb/2011aato/201212/ P020121210596715830424.pdf].

[4] Y. Zhao et al., Nucl. Instrum. Methods Phys. Res., Sect. B 267, 163 (2009).

[5] J. C. Yang et al., Nucl. Instrum. Methods Phys. Res., Sect. B 317, 263 (2013).

[6] P. Spiller, K. Blasche, B. Franczak, J. Stadlmann, and C. Omet, in Proceedings of 33rd ICFA Advanced Beam Dynamics Workshop on High Intensity and High Brightness Hadron Beams, Bensheim, Germany, 2004 (AIP, Bensheim, 2004), pp. 40-44.

[7] C Omet, P. Spiller, J. Stadlmann, and D. H. H. Hoffman, New J. Phys. 8, 284 (2006).

[8] P. Puppel, P. Spiller, L. Bozyk, and U. Ratzinger, in Proceedings of the International Particle Accelerator Conference, Kyoto, Japan, 2010 (ICR, Kyoto, 2010), pp. 594-596.

[9] L. Bozyk, P. Spiller, and H. Kollmus, in Proceedings of the International Particle Accelerator Conference,
New Orleans, Louisiana ,USA, 2012 (IEEE, Piscataway, NJ, 2012), pp. 3237-3239.

[10] J. W. Xia, W. L. Zhan, B. W. Wei, Y. J. Yuan, and M. T. Song, High Energy Phys. Nucl. Phys. 30, 335 (2006).

[11] J. W. Xia, in Proceedings of 4th Overseas Chinese Physics Association Accelerator School, Yanzhou, China, 2006 [http://ssrf.sinap.ac.cn/ocpaschools06/index.htm].

[12] Y. J. Yuan, in Proceedings of 52nd ICFA Advanced Beam Dynamics Workshop on High Intensity and High Brightness Hadron Beams, Beijing, China, 2012 (JACoW, Beijing, China, 2012), TU01B03.

[13] L. Bozyk (private communication).

[14] P. J. Bryant, in Proceedings of the 7th European Particle Accelerator Conference, EPAC 2000, Vienna, Austria (EPS, Geneva, 2000), pp. 1357-1359.

[15] S. Y. Lee, Accelerator Physics (World Scientific, Shang Hai, 2006), Vol. 2, p. 51.

[16] I. Meshkov, A. Sidorin, A. Smirnov, G. Trubnikov, and R. Pivin, BETACOOL Physics Guide [http://betacool.jinr.ru/ programs/Betacool\%20Physics\%20Guide.pdf].

[17] L. J. Mao, Ph.D. thesis, Graduate University of Chinese Academy of Sciences, 2008 [http://210.77.73.110/handle/ $113462 / 509$ ? mode $=$ full\&submit $\_$simple $=$Show + full + item +record].

[18] V. P. Shevelko, M.-Y. Song, I. Yu. Tolstikhina, H. Tawara, and J.-S. Yoon, Nucl. Instrum. Methods Phys. Res., Sect. B 278, 63 (2012).

[19] J. Pasternak, European Organization for Nuclear Research AB Report, AB-Note-2004-066.

[20] C. Omet, H. Kollmus, H. Reich-Sprenger, and P. Spiller, in Proceedings of the 11th European Particle Accelerator Conference, EPAC08, Genoa, Italy (EPS-AG, Geneva, Italy, 2008), pp. 295-297.

[21] R. S. Mao (private communication).

[22] J. Stadlmann, in Proceedings of CERN Collimator Workshop, (CERN, Geneva, 2007) [https://indico.cern.ch/event/ 15955/session/0/contribution/41/material/slides/1.pdf].

[23] Y. J. Yuan, H. W. Zhao, J. W. Xia, X. D. Yang, H. S. Xu, and CSR Group, in Proceedings of the Workshop on Beam Cooling and Related Topics (COOL09), Lanzhou, China, 2009 (JACoW, Lanzhou, 2009), pp. 185-189.

[24] L. Bozyk (private communication).

[25] R. S. Mao, Beam diagnostics at IMP [http://www-bd .gsi.deldokuwiki/lib/exelfetch.php?media=meetings:bdgroup_imp.pdf].

[26] Y. J. Yuan and J. W. Xia, in Proceedings of 8th International Conference on Nuclear Physics at Storage Rings (Storil1) (Laboratori Nazionali di Frascati dell'INFN, Italy, 2011), pp. 69-77.

[27] R. K. Kalil and F. E. Mills, Nucl. Instrum. Methods Phys. Res., Sect. A 346, 1 (1994).

[28] K. Y. Wei, Theory of Charged Particle Beam Transport (Scientific, Beijing, 1986), Vol. 14, p. 358.

[29] F. Christoph Iselin, Physical Methods Manual of the MAD Program [http://mad.web.cern.ch/mad/].

[30] G. Cai (private communication). 